\title{
ESPONDILITIS TUBERCULOSA EN ADULTOS: UNA SERIE DE CASOS EN UN HOSPITAL DE TERCER NIVEL, LIMA - PERÚ
}

\author{
Manuel Castillo-Angeles ${ }^{1, a}$, Celso De la Cruz Luque ${ }^{1, a}$, Henry Zelada $1, a$, \\ Ana Rosa Vilela-Sangay ${ }^{1, a}$, Frine Samalvides ${ }^{1,2, b}$, Germán Málaga ${ }^{1,3, c}$
}

\begin{abstract}
RESUMEN
Describimos las características clínicas y demográficas en pacientes adultos con espondilitis tuberculosa así como un análisis exploratorio que buscó establecer características que contribuyeron al desarrollo de esta enfermedad, en un hospital de tercer nivel de Lima. Realizamos un estudio tipo serie de casos y describimos 33 casos recolectados entre 1999-2009. 18 pacientes (55\%) fueron varones, la media de edad fue 31 años (IQ 23 a 51 años) y un tiempo de enfermedad de 3 meses (IQ 1 a 8 meses). El principal síntoma fue lumbalgia en 28 (85\%). Los segmentos más comprometidos fueron el torácico en $28(60 \%)$ casos y lumbar en $13(28 \%)$. Tuberculosis pulmonar se encontró en 14 (42\%) casos. Veinticuatro (73\%) recibieron esquema I. La duración de tratamiento fue 10,5 4,2 meses . Las características clínicas, diagnósticas fueron similares a series nacionales previas e internacionales.
\end{abstract}

Palabras clave: Espondilitis; Tuberculosis espinal; Mal de Pott (Fuente: DeCS BIREME).

\section{TUBERCULOUS SPONDYLITIS IN ADULTS: A CASE SERIES FROM A REFERENCE HOSPITAL IN LIMA, PERU}

\begin{abstract}
We describe the demographic and clinical characteristics of adult patients with tuberculous spondylitis as well as an exploratory analysis that seeked to establish patient characteristics that contributed to the development of this disorder at a reference hospital in Lima. This is a case-series descriptive study where data collection from 33 medical records of patients from 1999 to 2009 was done. Eighteen (55\%) were male. Median age was 31 years (IQR 23 to 51 years). Median time of symptoms was 3 months (IQR 1 to 8 months). The most frequent symptom was back pain in 28 (85\%). The most frequently affected areas were the thoracic spine involved in $28(60 \%)$ cases and lumbar spine in $13(28 \%)$. Pulmonary tuberculosis was present in fourteen $(42 \%)$ cases. Twenty four $(73 \%)$ patients received first line treatment. Treatment duration was $10.5 \pm 4.2$ months. Clinical and diagnostic characteristics were similar to previous Peruvian case reports and current literature.
\end{abstract}

Key words: Spondylitis; Tuberculosis, spinal; Pott disease (Source: MeSH NLM).

\section{INTRODUCCIÓN}

A pesar de que la incidencia de tuberculosis viene disminuyendo desde 1990 en nuestro país ${ }^{(1)}$, situaciones como el crecimiento poblacional acelerado, la migración no controlada, el persistente desempleo o subempleo y la emergencia de tuberculosis multidrogorresistente y extremadamente resistente sugerirían un cambio en las características epidemiológicas ${ }^{(2)}$ y hacen que se mantenga como un problema de salud pública. En el 2007, la incidencia en nuestro país fue de 116,5 casos por 100000 habitantes ${ }^{(1,3)}$.

La espondilitis tuberculosa (ET) es una forma de tuberculosis extrapulmonar que constituye el $1,7 \%$ de todos los casos de tuberculosis ${ }^{(4-6)}$, constituye el $50 \%$ de los casos de tuberculosis esquelética y, de estas, constituye la variante con mayor morbilidad debido a la destrucción ósea, deformidad y potencial déficit neurológico ${ }^{(7,8)}$. El desarrollo de mejores procedimientos diagnósticos no había disminuido el retraso para el diagnóstico especialmente en nuestro país donde $47 \%$ de ET eran diagnosticados alrededor de los seis meses ${ }^{(9,10)}$. Por tanto, la recomendación vigente es la de mantener un alto grado de sospecha clínica ${ }^{(1,2,9)}$, por el curso crónico indolente que caracteriza esta enfermedad ${ }^{(5-7,11,12)}$.

Existen pocos estudios sobre ET en nuestro medio y en la literatura internacional. Los últimos estudios en el Perú, fueron realizados hace más de una década; por lo que

\footnotetext{
1 Universidad Peruana Cayetano Heredia. Lima, Perú.

2 Departamento de Enfermedades Infecciosas y Dermatológica. Hospital Nacional Cayetano Heredia. Lima, Perú.

3 Departamento de Medicina. Hospital Nacional Cayetano Heredia. Lima, Perú.

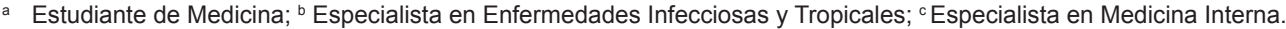


no incluyen las nuevas herramientas diagnósticas ni los nuevos esquemas de quimioterapia ${ }^{(9,10)}$. El proyecto describió y analizó de manera exploratoria las asociaciones entre variables características clínicas, diagnósticas y terapéuticas actuales de la ET en una serie de 33 casos.

\section{EL ESTUDIO}

Se realizó un estudio descriptivo, retrospectivo, tipo serie de casos. Los pacientes fueron identificados utilizando los registros de pacientes del Programa de Control de Tuberculosis (PCT) los que fueron correlacionados con los registros de egreso de un hospital de referencia de tercer nivel del cono norte de la ciudad de Lima (Hospital Nacional Cayetano Heredia). Se recabaron las historias clínicas de pacientes con diagnóstico de ET en el periodo enero 1999 a diciembre de 2009. Se registraron en fichas factores sociodemográficos, síntomas y su duración, factores predisponentes, formas de diagnóstico, laboratorio, serología para Brucella, HTLV-1 (human T-lymphotropic virus), VIH (virus de inmunodeficiencia humana), hallazgos radiológicos, resultados de estudios microbiológicos, hallazgos histopatológicos, tipos de terapia y respuesta a tratamiento.

Los casos fueron clasificados de acuerdo con los hallazgos clínicos y radiológicos compatibles con espondilitis, más el diagnóstico de tuberculosis por uno de los siguientes: a) evidencia microbiológica (bacilos ácido alcohol resistentes en coloración Ziehl Nielsen, cultivo positivo en medio Lowenstein-Jensen, reacción en cadena de polimerasa y test de adenosín deaminasa) de infección por tuberculosis de cualquier muestra (esputo, absceso, tejido óseo) ${ }^{(12,13) ; ~ b) ~ c a m b i o s ~ h i s t o p a t o l o ́ g i c o s ~}$ concordantes con tuberculosis; c) respuesta a terapia antituberculosa (mejoría clínica o regresión de los hallazgos radiológicos) en ausencia de aislamiento de $M y$ cobacterium tuberculosis ${ }^{(9,12)}$. Los criterios de exclusión fueron: datos incompletos en la historia clínica (falta de informes de los estudios de imágenes realizados).

Los esquemas de tratamiento usados en estos 10 años fueron el esquema I del PCT: régimen de cuatro drogas por 12 semanas por vía oral (isoniazida $5 \mathrm{mg} / \mathrm{kg} / \mathrm{d}$, rifampicina $10 \mathrm{mg} / \mathrm{kg} / \mathrm{d}$, etambutol $15 \mathrm{mg} / \mathrm{kg} / \mathrm{d}$ pirazinamida $25 \mathrm{mg} / \mathrm{kg} / \mathrm{d}$ ), seguido de isonicida y rifampicina por ocho meses; el esquema II del del PCT implicó, agregar estreptomicina $15 \mathrm{mg} / \mathrm{kg} / \mathrm{d}$ intramuscular y el esquema estandarizado se empleo como se recomienda, de acuerdo con los resultados de susceptibilidad. La duración del tratamiento dependió, en su momento, de la decisión del médico tratante que evaluó factores como la respuesta a dicho tratamiento y mejoría clínica y radiológica. La respuesta al tratamiento fue consignada en forma subjetiva de los datos descritos de la evolución de los pacientes que se encontraban en las historias clínicas.

Los resultados fueron presentados con medidas de tendencia central y sus respectivas medidas de dispersión. Se buscó asociación entre las variables del estudio para lo cual se utilizó el programa STATA versión 10 para el análisis estadístico. El test de $U$ de Mann-Whitney se utilizó para las variables continuas y el test de $x^{2}$ para variables categóricas. Una $p<0,05$ se consideró como significativo.

\section{HALLAZGOS}

Se obtuvo 41 historias durante el período 1999 - 2009 . Se seleccionaron 33 casos (Tabla 1), cinco no tenían confirmación diagnóstica y tres no tenían informe de estudios de imagen. De los 33 casos, 18 (55\%) eran varones (Tabla 2). La edad de estos casos no seguía una distribución normal, obteniendo una mediana de 31 años con rango intercuartil de 23 a 53 años. El rango de edad obtenido fue 18 a 77 años.

\section{CUADRO CLÍNICO}

El tiempo de enfermedad tuvo una mediana de tres meses, con rango intercuartil de uno a ocho meses. El número de consultas previas tuvo una media de 1,7. Entre los síntomas, lumbalgia estuvo presente en 28 pacientes (85\%), mientras manifestaciones sistémicas, alteración sensitiva e impotencia funcional estuvieron en 20 casos $(61 \%)$. La comorbilidad más frecuente fue la presencia de otros órganos o sistemas afectados por la enfermedad por tuberculosis en 17 casos (52\%) de los cuales 14 pacientes $(42 \%)$ presentaron compromiso pulmonar en forma concomitante con el compromiso esquelético; de ellos seis pacientes presentaron un baciloscopía de esputo positiva. No se observo coexistencia con otros tipos de tuberculosis esquelética. Se detectaron tres casos de inmunosupresión, de ellos, dos (6\%) portadores diabetes mellitus tipo 2 y uno portador de lupus eritematoso sistémico (3\%). Las pruebas rápidas para infección por Virus de Inmunodeficiencia Humana (VIH) fueron realizadas en todos los pacientes, siendo todas negativas. El tratamiento previo más utilizado fue antinflamatorios no esteroideos (AINES) en 11 casos (33\%) y la asociación AINES más corticoides se utilizo en seis pacientes (18\%). El corticoide empleado en todos los casos fue dexametasona.

\section{DIAGNÓSTICO}

La confirmación del diagnóstico fue bacteriológica en 15 casos $(45 \%)$, de los cuales seis fueron por hallazgo 
Tabla 1: Características de los casos incluidos.

\begin{tabular}{|c|c|c|c|c|c|c|c|c|c|c|}
\hline $\begin{array}{c}\mathrm{N}^{\circ} \\
\text { paciente }\end{array}$ & Edad & Género & $\begin{array}{l}\text { Tiempo Enf } \\
\text { (días) }\end{array}$ & Historia TBC & VSG (mm/h) & PPD (mm) & $\begin{array}{c}\text { Segmento } \\
\text { comprometido }\end{array}$ & Destrucción & Absceso & Compresión \\
\hline 1 & 19 & Femenino & 365 & No & NR & Negativo & Dorsal & Sí & Sí & No \\
\hline 2 & 55 & Masculino & 14 & No & 55 & 13 & Dorsal & Sí & No & Sí \\
\hline 3 & 52 & Masculino & 90 & Pulmonar & NR & NR & Dorsal & Sí & Sí & Sí \\
\hline 4 & 18 & Masculino & 90 & No & NR & Negativo & Dorsal & No & No & No \\
\hline 5 & 26 & Masculino & 30 & No & 50 & NR & Dorsolumbar & Sí & Sí & No \\
\hline 6 & 42 & Femenino & 180 & Renal & NR & NR & Lumbar & No & No & Sí \\
\hline 7 & 25 & Femenino & 21 & Pumonar & 145 & 15 & Dorsolumbar & Sí & Sí & No \\
\hline 8 & 23 & Femenino & 60 & Pulmonar & NR & 20 & Dorsal & Sí & No & No \\
\hline 9 & 28 & Masculino & 30 & Pulmonar & 28 & NR & Lumbar & Sí & Sí & No \\
\hline 10 & 36 & Masculino & 180 & Renal & NR & 20 & Dorsolumbar & No & Sí & No \\
\hline 11 & 72 & Masculino & 330 & Pulmonar & NR & NR & Dorsolumbar & Sí & No & No \\
\hline 12 & 65 & Masculino & 180 & Pulmonar & NR & Negativo & Dorsolumbar & No & No & No \\
\hline 13 & 77 & Femenino & 120 & Pulmonar & 0 & 10 & Dorsal & No & No & No \\
\hline 14 & 54 & Masculino & 30 & No & 50 & NR & Lumbar & Sí & No & No \\
\hline 15 & 18 & Masculino & 60 & No & 55 & NR & Lumbar & No & No & No \\
\hline 16 & 51 & Femenino & 240 & No & 30 & NR & Dorsal & Sí & No & No \\
\hline 17 & 35 & Femenino & 180 & Pulmonar & 37 & NR & Dorsal & Sí & No & No \\
\hline 18 & 29 & Masculino & 60 & Pulmonar & NR & NR & Dorsal & Sí & Sí & No \\
\hline 19 & 27 & Femenino & 21 & Pulmonar & NR & NR & Dorsal & No & Sí & No \\
\hline 20 & 26 & Masculino & 30 & No & 50 & NR & Dorsolumbar & Sí & Sí & Sí \\
\hline 21 & 49 & Masculino & 30 & Renal & NR & NR & Dorsal & No & No & No \\
\hline 22 & 18 & Femenino & 22 & No & NR & Negativo & Dorsolumbar & Sí & No & No \\
\hline 23 & 53 & Femenino & 240 & No & 37 & NR & Lumbar & Sí & No & No \\
\hline 24 & 18 & Masculino & 60 & No & 30 & NR & Dorsal & Sí & No & No \\
\hline 25 & 19 & Masculino & 2190 & No & 24 & NR & Lumbar & Sí & No & No \\
\hline 26 & 20 & Femenino & 575 & No & NR & 13 & Dorsal & Sí & No & No \\
\hline 27 & 31 & Femenino & 180 & Pulmonar & NR & NR & Dorsolumbar & Sí & Sí & No \\
\hline 28 & 21 & Masculino & 485 & Pulmonar & 38 & NR & Lumbar & Sí & Sí & No \\
\hline 29 & 56 & Masculino & 30 & Pulmonar & NR & Negativo & Dorsal & Sí & No & No \\
\hline 30 & 23 & Femenino & 90 & No & NR & 19 & Dorsal & Sí & No & No \\
\hline 31 & 67 & Femenino & 390 & No & 78 & 22 & Lumbar & No & Sí & No \\
\hline 32 & 62 & Femenino & 60 & Pulmonar & 49 & 22 & Dorsal & Sí & No & Sí \\
\hline 33 & 43 & Masculino & 365 & No & NR & NR & Lumbar & Sí & Sí & No \\
\hline
\end{tabular}

microbiológico, seis por hallazgo patológico y tres por ambos (Tabla 3). Se realizaron trece biopsias, diez de cuerpo vertebral y tres de abscesos paravertebrales. De ellas, nueve (69\%) mostraron necrosis caseosa, los cuatro restantes solo hallazgos inflamatorios inespecíficos. Solo 1/6 muestras enviadas para cultivo de BK resultó positiva. No se realizó ninguna prueba de adenosina deaminasa $(A D A)$ o reacción en cadena de polimerasa en el grupo estudiado.

Tabla 2. Características clínicas de casos.

\begin{tabular}{lc}
\hline Variables & Resultados \\
\hline Edad (años) & $31($ IQ 23-53) \\
Sexo Masculino & $18(55 \%)$ \\
Tiempo de Enfermedad (meses) & $3($ IQ $1-8)$ \\
Segmento dorsal (\%) & $16(49 \%)$ \\
Segmento lumbar (\%) & $9(28 \%)$ \\
Dorsolumbar (\%) & $8(26 \%)$ \\
Tratamiento quirúrgico & $11(33 \%)$ \\
Colapso (\%) & $24(73 \%)$ \\
Compresión (\%) & $13(39 \%)$ \\
Absceso (\%) & $4(12 \%)$ \\
\hline
\end{tabular}

\section{EXÁMENES AUXILIARES}

En el laboratorio, la media de velocidad de sedimentación globular fue $46,7 \mathrm{~mm} / \mathrm{hr}$ con desviación estándar de 32,7 . Nueve (64\%) de 14 pacientes tuvieron prueba de Mantoux (PPD) positiva. En diecinueve pacientes

Tabla 3. Confirmación diagnóstica de casos.

\begin{tabular}{lc}
\hline & $\mathbf{n}$ \\
\hline A) Criterio microbiológico & \\
BK esputo & 2 \\
BK absceso & 3 \\
Cultivo BK & 1 \\
ADA & 0 \\
$\quad$ Reacción cadena polimerasa & 0 \\
Total & 6 \\
B) Criterio patológico & \\
$\quad$ Necrosis caseosa & 6 \\
$\quad$ A + B & \\
$\quad$ Biopsia + BK esputo & 3 \\
Total & 3 \\
C) Respuesta tratamiento & 18 \\
Total & 33 \\
\hline
\end{tabular}




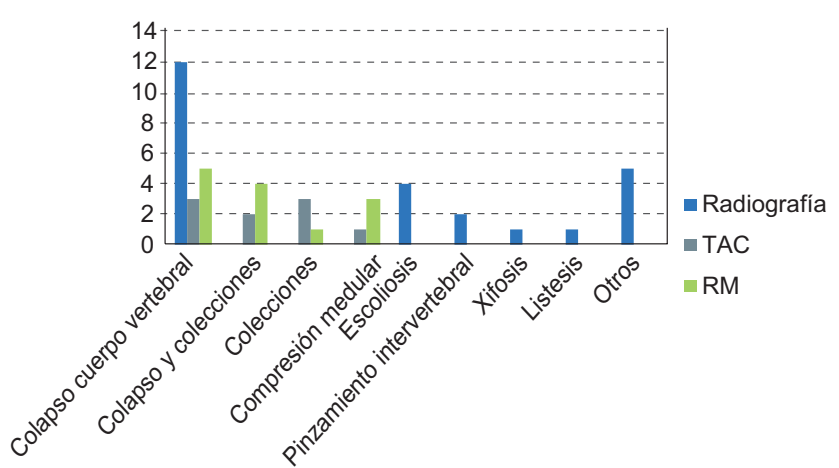

Figura 1. Hallazgos radiológicos según tipo de imágenes.

se realizó serología de Brucella, todos fueron negativos.

Hallamos compromiso de columna dorsal en 16 (46\%) de imágenes, dorsolumbar en seis casos (18\%) y lumbar en once casos (36\%). Se realizaron 47 imágenes en total. De estas, 24 fueron radiografías de columna vertebral; el hallazgo más común fue colapso de cuerpo vertebral en $12(50 \%)$ radiografías, mientras en cuatro (17\%) se describió escoliosis. Se tomaron nueve tomografías, se describió colecciones paravertebrales en tres casos. Se tomaron 14 resonancias magnéticas, lo hallado más común fue colapso vertebral en cinco casos (36\%) y compresión medular en tres casos (21\%) (Figura 1).

Los segmentos comprometidos fueron D11 con 13 (46\%) de 28 imágenes, seguido de D8 con seis (21\%), D6 con cuatro $(14 \%)$, D5 con tres $(11 \%)$ y D7 con dos $(8 \%)$ imágenes. El compromiso lumbar se registró en 13 (28\%) de imágenes; los segmentos comprometidos fueron L4 con siete imágenes y L3 con seis. Hubo compromiso dorso lumbar en seis imágenes, describiéndose en todas un compromiso desde D11 hasta L1.

\section{TRATAMIENTO}

Con relación al tratamiento farmacológico, 24 (73\%) pacientes iniciaron con esquema I, seis (18\%) iniciaron con esquema II y tres (9\%) recibieron esquema estandarizado. La media de duración de tratamiento farmacológico fue 10,5 meses con desviación estándar de 4,2. Once (33\%) pacientes, además, recibieron tratamiento quirúrgico. La técnica más común fue laminectomía en cuatro pacientes y desbridamiento en tres casos. Las indicaciones para cirugía fueron déficit neurológico en cuatro pacientes, drenaje de absceso en otros cuatro, compromiso severo de cuerpo vertebral en dos y falla a tratamiento médico en un paciente. En cuanto a las secuelas, en cinco $(15 \%)$ pacientes se registro vejiga neurogénica, tres (9\%) paraplejía y dos (6\%) tuvieron dificultad para la marcha. No se registró ningún fallecimiento.
Se exploró las siguientes asociaciones: sexo de los pacientes se relaciono con tipo de tratamiento recibido y con los distintos esquemas de tratamiento. Además, se relacionó tiempo de enfermedad con duración del tratamiento y presencia de comorbilidades con duración de tratamiento. En ninguna de las asociaciones exploradas se obtuvo diferencia significativa.

\section{DISCUSIÓN}

Nuestra mediana de edad (31 años con intervalo de 23 a 53 años); así como la predominancia de sexo masculino, es similar a series nacionales previas, donde la media fue 27 años ${ }^{(8,9)}$.

Un hallazgo interesante que resaltar fue el menor tiempo transcurrido entre el inicio de los síntomas y el diagnóstico, que ocurrió alrededor de los tres meses, tiempo menor que en series nacionales previas e internacionales donde se describe medias de aproximadamente diez meses con desviación estándar $11,8^{(8,9,10,14)}$, así como el reducido número de consultas antes de lograr el diagnóstico. Estas diferencias sugieren que en nuestro medio la ET es considerada con frecuencia, como diagnóstico diferencial.

El síntoma más común reportado, fue lumbalgia, en 28 $(85 \%)$ casos, coincidiendo con lo descrito en series internacionales ${ }^{(8,13)}$; seguido de manifestaciones sistémicas y déficit neurológico que se presentaron en 20 casos (61\%). Esto sugiere que la presencia de lumbalgia asociado a manifestaciones sistémicas o neurológicas, debe hacer considerar ET dentro de las opciones diagnósticas.

La columna vertebral torácica fue el segmento más comprometido en imágenes, lo que es coincidente con series nacionales e internacionales ${ }^{(9,10,13,14)}$. Esta aparente discordancia entre el síntoma más común que fue lumbalgia y el compromiso más común que fue la afectación de la columna vertebral dorsal, podría explicarse, dado que al ser el segmento más comprometido D11, el dolor referido de dicha zona pueda registrarse como lumbalgia en la mayoría de casos o a que nuestros pacientes refieren genéricamente como "lumbalgia" a las manifestaciones de la "espalda baja". A su vez, el hallazgo de colecciones paravertebrales, hallazgo característico en ET a diferencia de otras espondilitis, estuvo presente en diez $(23 \%)$ de las imágenes obtenidas.

Nos llamó la atención la relativamente baja proporción de pacientes en quienes se completaron estudios tomográficos, de RNM y biopsias, lo que podría haber mostrado mejores porcentajes de confirmación diagnóstica 
resultados, más acordes con lo reportado en series internacionales.

Un aspecto llamativo es la variabilidad de los esquemas terapéuticos utilizados, si bien la mayoría recibió esquema I (23 casos), esta no se cumplió estrictamente en lo relacionado con la duración del tratamiento, pues como vimos, la media de duración del tratamiento farmacológico fue de 10,5 meses $\pm 4,2$ ). Estos hallazgos sugieren que a pesar de los éxitos del PCT, aún no se ha logrado consenso para el esquema terapéutico apropiado de algunas formas de tuberculosis extrapulmonar como ET.

Un hallazgo interesante lo constituye el uso de corticides, que ocurrió en todos los casos, a pesar de que no hemos hallado estudios que sustenten su uso en pacientes con ET.

A su vez, once pacientes (33\%) fueron sometidos a intervención quirúrgica, las indicaciones para cirugía fueron déficit neurológico en cuatro pacientes, drenaje de absceso en otros cuatro, compromiso severo de cuerpo vertebral en dos y falla a tratamiento médico en un paciente; sin embargo, no podemos afirmar que exista un criterio uniforme para realizar este tipo de intervenciones y corresponderían a decisiones personales no protocolizadas. El porcentaje de pacientes intervenidos es similar a una serie nacional previa donde 13 de 45 (29\%) casos fueron a cirugía; pero es menor al 85 - 90\% descritos en series internacionales ${ }^{(8,15)}$. Esto se explica por la dificultad de nuestra población hospitalaria para acceder económicamente a tratamiento neuroquirúrgico, indicado en etapas avanzadas de la enfermedad o por falla del tratamiento médico ${ }^{(8,12,15)}$. Cabe resaltar dos cosas: primero, en una serie de Taiwan no se halla diferencias significativas en cuanto a tratamiento quirúrgico y tratamiento médico ${ }^{(13)}$, pero tiene el limitante de que su muestra es pequeña, por lo que la diferencia pudo no haber sido constatada. Segundo, el último estudio experimental donde se comparó tratamiento médico frente a tratamiento quirúrgico mostró mayor beneficio en la terapia médica, pero esa muestra incluye población pediátrica y una proporción de compromiso neurológico ${ }^{(16)}$ distinta a nuestra serie, por lo que sus resultados no son directamente aplicables.

Los hallazgos que reportamos referidos a secuelas neurológicas y mortalidad, son similares a los reportados en series nacionales e internacionales previas a pesar del relativamente bajo acceso a tratamiento quirúrgico y rehabilitación, podría sugerir que el reconocimiento precoz, así como que la baja tasa de falla a tratamiento farmacológico de ET, es baja en nuestro centro hospitalario e influirían en este relativo buen resultado. A su vez, esta evolución sugeriría que ni la aparición de tuberculosis multidrogorresistente (MDR), ni el aumento de casos de VIH han influido en cambios de presentación de esta enfermedad.

La fortaleza del estudio es el número de casos recolectado a pesar de ser una entidad poco frecuente, lo que permite tener un panorama interesante de las características clínicas y epidemiológicas en nuestro medio. Las limitaciones del estudio incluyen que no todos los pacientes tuvieron acceso a todas las modalidades diagnósticas y terapéuticas, lo cual no ha variado en relación a series previas. Además no se utilizó escala funcional para medición de secuelas y no había registro de severidad inicial en las historias clínicas evaluadas, impidiendo correlacionar severidad inicial con secuelas. A su vez, el diseño de serie de casos solo permite explorar asociaciones entre variables y sugerir hipótesis. El carácter retrospectívo de nuestra serie también es un factor a considerar como posible fuente de sesgos.

Finalmente la ET parece no haber sufrido mayores variaciones clínicas a través del tiempo en nuestro centro y se ajusta a los hallazgos de series internacionales a excepción de la menor media de edad y el menor tiempo de enfermedad posiblemente gracias a un diagnóstico más temprano.

\section{Contribuciones de Autoría}

Todos los autores participaron en la concepción del trabajo de investigación, en la elaboración del manuscrito, en su revisión crítica y aprobación del documento final.

\section{Fuentes de Financiamiento}

Autofinanciado.

\section{Conflictos de Interés}

Los autores declaran no tener conflictos de interés.

\section{REFERENCIAS BIBLIOGRÁFICAS}

1. Bonilla C. Situación de la tuberculosis en el Perú. Acta Med Per. 2008;25(3)163-70.

2. Becerra M, Appleton S, Franke M, Chalco K, Arteaga F. Tuberculosis burden in households of patients with multidrug-resistant and extensively drug-resistant tuberculosis: a retrospective study. Lancet. 2011;377(9760):147-52.

3. Organización Mundial de Salud; Organización Panamericana de Salud Tuberculosis en las Américas Reporte Regional 2009 HSD/CD/T/002-10. Washington, DC: OPS; 2009.

4. Fitzgerald D, Haas DW. Mycobacterium tuberculosis. In: Mandell GL, Bennett JE, Dolin R, eds. Mandell, Douglas and Bennett's Principles and Practice of Infectious Diseases. $6^{\text {th }}$ ed. Philadelphia, PA: Elsevier Churchill Livingstone; 2005. p. 2852-85. 
5. García del Pozo EJ. Espondilitis infecciosa: Estudio clínico y valoración de secuelas funcionales [tesis doctoral]. Madrid: Universidad Autónoma de Madrid; 2003.

6. Loja D, Vilca M, Avil R, N Yngrid, Carrasco J. Mal de Pott: Diagnostico y tratamiento. Bol Soc Per Med Int. 1999;12(3):8-16.

7. De la Torre D, Gongora-Lopez J, Huerta V, Perez-Maeve J. Enfermedad de Pott. Un reto diagnostico y terapéutico para el médico actual. Cir Ciruj. 2000;68(3):108-13.

8. Tasova Y, Sarpel T, Seza A, Saltoglu N, Guzel R, Kurtaran B. A Clinical Review of 40 cases with Tuberculous Spondylitis in Adults. Neurosurg Q. 2006;16(4):169-75.

9. Figueroa, $\mathrm{O}$ Tuberculosis osteoarticular: estudio retrospectivo de 105 pacientes en el Hospital General Base Cayetano Heredia. Lima. [tesis de especialidad]. Lima: Universidad Peruana Cayetano Heredia; 1983.

10. Ugarte, C. Tuberculosis osteoarticular en el Hospital Nacional "Edgardo Rebagliati Martins" (HNERM). Lima. [tesis de especialidad]. Lima: Universidad Peruana Cayetano Heredia; 1987.

11. Alvarez A. Hospitalización por causa reumatológica: 20 años de experiencia en el Hospital Nacional Cayetano Heredia 1970-1989. Lima [tesis de especialidad]. Lima: Universidad Peruana Cayetano Heredia; 1991.

12. Safo S, Lieberman G. Pott's disease: A Radiological Review of Tuberculous Spondylitis. Boston: Harvard Medical School; 2009
13. Shou-Hsin S, Wen-Cheng T, Chun-Yu L, Wei-Ru L, Tun-Chieh Chen, Po-Liang $L$ et al. Clinical Features and Outcomes of Spinal Tuberculosis in Southern Taiwan. J Microbiol Immunol Infect. 2010;43(4):291-300.

14. van Loenhout-Rooyackers JH, Verbeek AL, Jutte PC. Chemotherapeutic treatment for spinal tuberculosis. Int $\mathrm{J}$ Tuberc Lung Dis. 2002;6:259-65.

15. Turgut M. Multifocal extensive spinal tuberculosis (Pott's disease) involving cervical, thoracic and lumbar vertebrae. British Journal of Neurosurgery. 2001;15(2):142-6.

16. Parthasarathy $R$, Sriram $K$, Santha $T$, Prabhakar $R$, Somasundaram PR, Sivasubramanian S. Short-course chemotherapy for tuberculosis of the spine. A comparison between ambulant treatment and radical surgery--ten-year report. J Bone Joint Surg Br. 1999;81(3):464-71.

Correspondencia: Germán Málaga Rodríguez.

Dirección: Calle Chavín 159 CC Monterrico, Lima 33, Perú. Teléfono: (511) 992-768300

Correo electrónico: gmalaga01@gmail.com

\section{Consulte la versión electrónica de la Revista Peruana de Medicina Experimental y Salud Pública en}

\section{wWW.scopus.com}

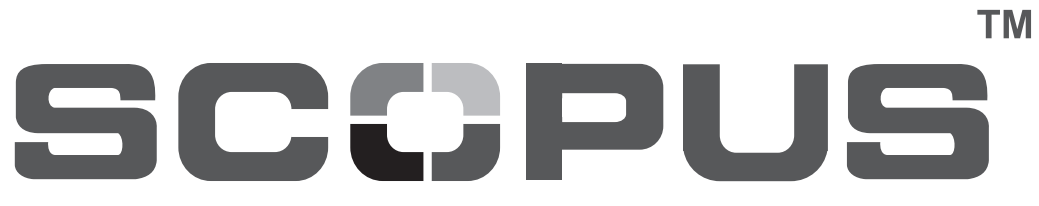

